\title{
An unusual cause of heart failure in a young patient
}

\begin{abstract}
The three Valsalva sinuses are hollow structures named after the corresponding aortic valve cusps. Valsalva sinus aneurysms are relatively rare $(0.14-0.23 \%)$, congenital or acquired, the most common being congenital, most often affecting the right coronary sinus. They also may be secondary to bacterial endocarditis, syphilis, atherosclerosis, aortic dissection, Marfan syndrome or as a result of severe chest trauma. Men's ratio: women is 4:1.

The Valsalva sinus aneurysm may be caused by cystic medial necrosis and the aortic annulus ectasia. The aneurysm can comunicate with a heart cavity, usually the right atrium (RA) or right ventricle(RV) near the membranous septum. The aneurysm rupture, which is usually asymptomatic, can create a communication with the right heart which permits a large left to right shunt with the installation of heart failure. Often the aneurysm concerns the right coronary sinus (in approximately $70 \%$ of cases), and the rupture occurs in this case usually in the RV and less frequently in the RA. The non-coronary sinus is less often involved (20$24 \%$ ) and it perforates more frequently in the RA. They can compress coronary arteries causing myocardial ischemia, may associate aortic regurgitation and may rupture into right cardiac cavities with acute heart failure symptoms. The rupture into pericardium leads to cardiac tamponade and death. The natural evolution is based on the size and etiology of the aneurysm, with increased risk of rupture.
\end{abstract}

Keywords: valsalva sinus aneurysm, cardiac heart failure, left to right shunt
Volume 9 Issue I - 2017

\author{
Andreea Taisia Tiron, Daniela Stegaru, \\ Gheorghita Aron \\ Department of Cardiology, ?Sf. loan? Emergency Hospital, \\ Romania
}
Correspondence: Andreea Taisia Tiron, Department of Cardiology, $\square$ Sf. loan $\square$ Emergency Hospital, Soseaua Vitan Barzesti no 13, Bucharest, Romania,

Email taisia_andreea@yahoo.com

Received: May II, 2017 | Published: June 12, 2017

\section{Introduction}

The Valsalva sinus aneurysms are a rare pathology, most often congenital but also secondary to bacterial endocardites, syphilis, atherosclerosis, aortic dissection, chest trauma, Marfan syndrome, with higher incidence in men. The non-coronary sinus is less oftern involved and usually it perforates into the right atrium with appearance of heart failure due to the left to right heart shunt. The rupture of the aneurysm is usually asymptomatic. The diagnosis is made by echocardiographic examination. The medical treatment is the treatment of the heart failure, arrhythmia, bacterial endocardites. The cure is surgical closure of the perforation and reconstruction of the aortic wall or aortic valve repair/replacement.

\section{Case Summary}

33 years old male patient, smoker, with a history of treated asthma , left posttraumatic pneumothorax with repeted pleurodesis, solved by surgery is admitted for dyspnea at rest, dry cough accentuated in clinostatism and cyanosis, symptoms that worsened two months before admission, onset two years ago. The clinical examination revealed a left latero-thoracic postsurgical scar, mixt cyanosis, rest dyspnea, dry cought, normal pulmonary auscultation, $\mathrm{SpO} 2=97 \%$, anterior chest pain, turgid jugular veins, tachycardia, $\mathrm{BP}=130 / 70 \mathrm{mmHg}$, left parasternal continuous murmur of high intensity, accentuated by Valsalva maneuver, loud S 2 palpable in pulmonary area, systolic tricuspid murmur, no edemas, hepatomegaly and hepato-jugular reflux. Electrocardiogram (Figure 1) showed sinusal tachycardia, 120bpm, right bundle branch block, right axis deviation. The blood samples revealed myocardial and hepatic cytolyse, increased creatinine. On chest X-ray as showed in Figure 2 we found cardiomegalia, straight left middle cardiac arch, pulmonary stasis, latero-basal pachypleurites and left fissure inflamation. Performing the transthoracic ecocardiography (Figure 3a-3c) we noted normal LV systolic function, enlargement of the right cavities and pulmonary artery, mild mitral regurgitation, medium-mild pulmonary regurgitation, moderate functional tricuspidian regurgitation, noncoronary Valsalva sinus aneurysm ruptured into the RA with hemodynamic significant left to right shunt and mild pericardites. The contrast echocardiography found slow opacification of the right cavities without comunication between right and left cavities. During Valsalva maneuver on echocardiography air bubbles are visible into the right cavities with dissapearance after 10minutes of left lateral decubitus, during this event the patient's dyspnea becomes more intense. The thoracic CT scan fails to find a broncho-vascular fistula. The transesophageal echocardiography better defines the communication without other congenital defects. Abdominal ultrasound reveales mild ascites and hepatosplenomegalia. $\mathrm{He}$ receives treatment with antialdosteronic and loop diuretic, unfractioned heparine, beta blockers, bronchodilator and intermitent oxygenoterapy. The patient is transferred for the surgical repair with good evolution and hemodynamic compensation. During surgery no other defects or fistulas were identified. On follow-up the patient was stable with mild pulmonary hypertension, one episode of atrial flutter and no signs of heart failure. ${ }^{1-3}$

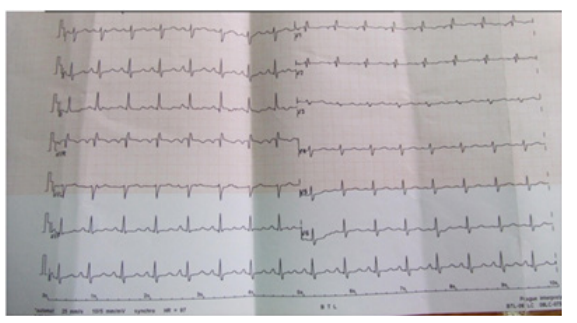

Figure I Coronary angiogram in LAO Cranial view of the right coronary artery showing normal coronary.

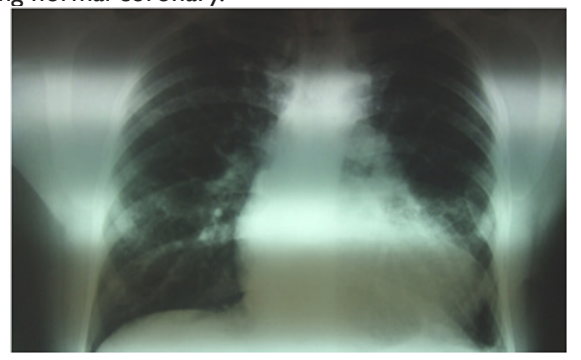

Figure $\mathbf{2}$ Thoracic asymetria due to scoliosis, cardiomegalia, pulmonary stasis, dilatation of pulmonary artery, pleural fibrosis. 


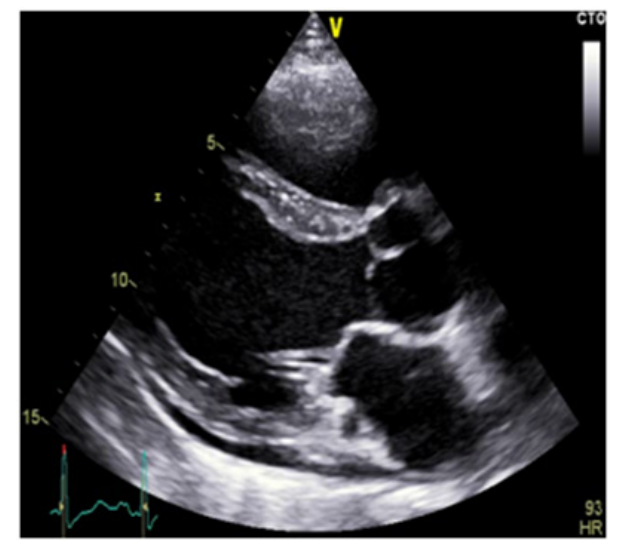

Figure 3a LV hypertrophy with normal function, asymmetry of the aortic cusps, dilated coronary sinus, mild pericardial effusion, normal mitral valve.

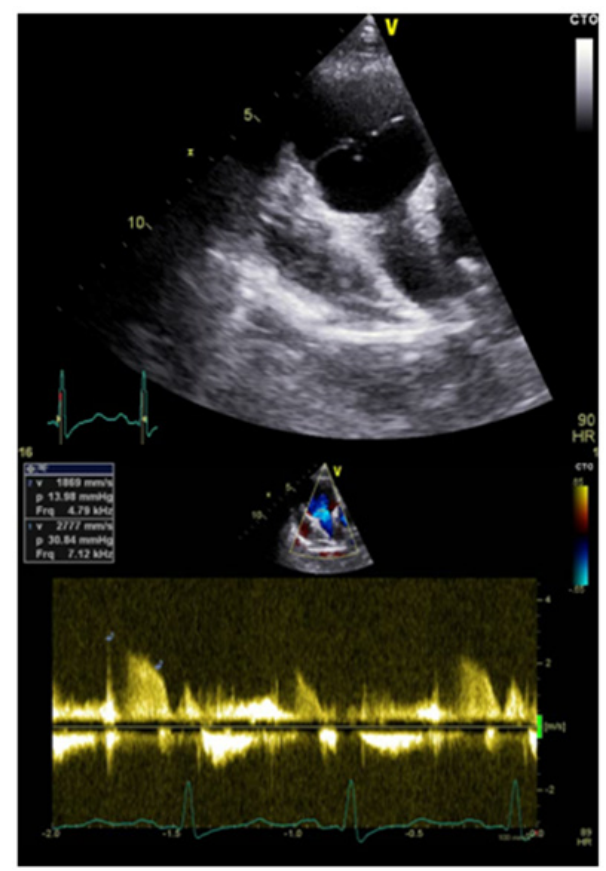

Figure 3b Pulmonary artery dilatation with mild regurgitation and pulmonary hypertension, $\mathrm{PAPm}=32 \mathrm{mmHg}$.
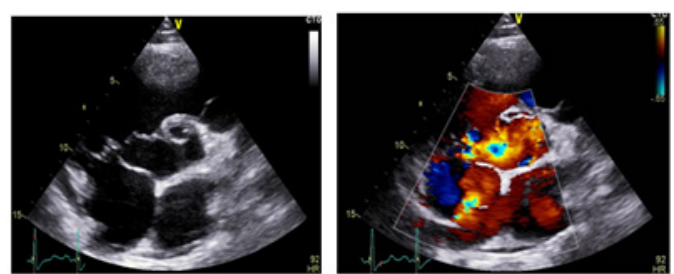

Figure 3c Tricuspid aorta, non-coronary sinus aneurysm communicating with the RA, left to right shunt; mild aortic regurgitation.

\section{Discussion}

Most of the Valsalva sinus aneurysms are congenital and associated with structural anomalies like ventricular sept defect, aortic bicuspidia, aortic regurgitation. In this case the rupture of the aneurysm might have been caused by the previous thoracic trauma. As in this case the rupture is described to be more often in the $3^{\text {rd }}$ of $4^{\text {th }}$ decade of life with evolution toward pulmonary hypertension and heart failure. The presence of arterial pulmonary hypertension is in favour of previous rupture and slow evolution of disease with left to right shunt until the appearance of heart failure symptoms that made the patient to come to emergency room. Early diagnosis and surgical repair, before arterial pulmonary hypertension appearance, is associated with a better long term prognostic.

\section{Acknowledgements}

Special thanks to Proff. Bogdan Popescu, cardiologist and Proff. Horatiu Moldovan, cardiac surgeon from the Emergency Cardiovascular Disease Institute „C. C. Iliescu”, Bucharest, Romania.

\section{Conflicts of interest}

Author declares there are no conflicts of interest.

\section{Funding}

None.

\section{References}

1. Meier JH, Seward JB, Miller FA, et al. Aneurysms in the left ventricular outflow tract: clinical presentation, causes, and echocardiographic features. J Am Soc Echocardiogr. 1998;11(7):729-745.

2. Ring WS. Congenital Heart Surgery Nomenclature and Database Project: Aortic Aneurysm, Sinus of Valsalva Aneurysm, and Aortic Dissection. Ann Thorac Surg. 2000;69(4 Suppl):S147-S163.

3. Wang KY, St John Sutton M, Ho HY, et al. Congenital sinus of Valsalva aneurysm: a multiplane transesophageal echocardiographic experience. $J$ Am Soc Echocardiogr. 1997;10(9):956-963. 